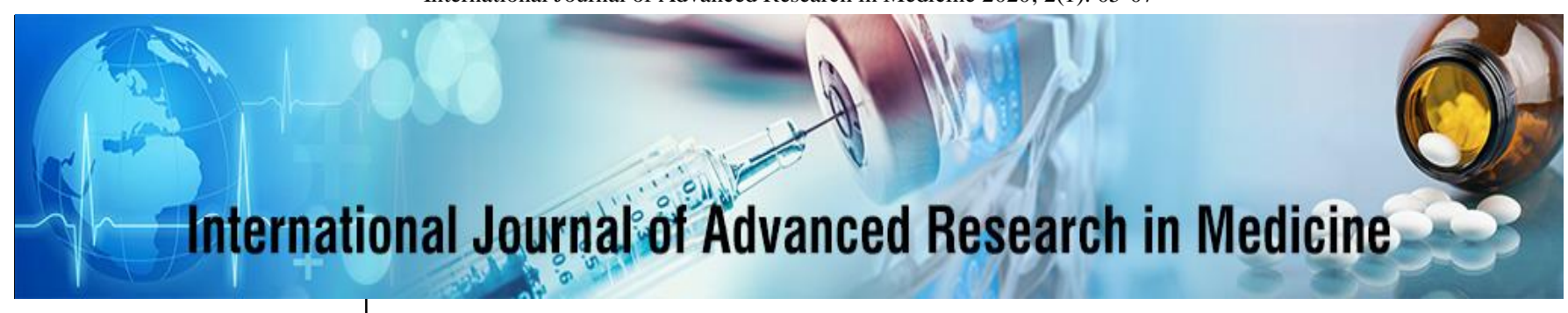

E-ISSN: 2706-9575 P-ISSN: 2706-9567 IJARM 2020; 2(1): 05-07 Received: 02-01-2020 Accepted: 03-02-2020

Dr. Mohd Akram Qureshi MD Pulmonary Medicine, Bidar Institutes of Medical Science, Bidar, Karnataka, India

Corresponding Author: Dr. Mohd Akram Qureshi MD Pulmonary Medicine, Bidar Institutes of Medical Science, Bidar, Karnataka, India

\section{Spectrum of pulmonary disease affecting the persons admitted to respiratory intensive care unit}

\author{
Dr. Mohd Akram Qureshi \\ DOI: https://doi.org/10.22271/27069567.2020.v2.i1a.29
}

\begin{abstract}
Background: Intensive care may be broadly defined as a service for patients who have potentially recoverable conditions, who can benefit from more detailed observation and invasive treatment that can be provided safely in a high dependency area. Respiratory ICU patients are a heterogeneous group with severe illness, multiple system dysfunction, and multiple coexisting medical problems.

Aim of the study: To study spectrum of pulmonary disease affecting the persons admitted to respiratory intensive care unit.

Materials and methods: The present study was conducted in the Department of Pulmonary Medicine of the Medial institute. The ethical approval for the study was approved from the ethical board of the institute before starting the study. For a study, a total of 100 patients with various respiratory diseases admitted to pulmonary ICU were selected. A written informed consent was obtained from everyone after verbally explaining them the study protocol. A complete demographic data for each patient was obtained including past medical history, past surgical history, family history, social history, history of present illness.

Results: We observed that mean age of the participants was 51.65 years. The age of participating patients ranged between 30 to 70 years. The number of male subjects was 66 and number of female subjects was 34 . We observed that highest number of patients had bronchial asthma $(n=22)$, followed by chronic eempyema $(n=19)$, Pulmonary Tb $(n=16)$, COPD $(n=14)$, Pneumothorax $(n=12)$, pneumonia $(\mathrm{n}=11)$.

Conclusion: Within the limitations of the present study, it can be concluded that bronchial asthma, chronic eempyema, Pulmonary $\mathrm{Tb}, \mathrm{COPD}$ and Pneumothorax are most common disease among pulmonary admitted patients.
\end{abstract}

Keywords: pulmonary ICU, asthma, COPD, pneumonia

\section{Introduction}

Intensive care may be broadly defined as a service for patients who have potentially recoverable conditions, who can benefit from more detailed observation and invasive treatment that can be provided safely in a high dependency area. Intensive care represents the highest level of continuing patient care and treatment ${ }^{[1-3]}$. Respiratory ICU patients are a heterogeneous group with severe illness, multiple system dysfunction, and multiple coexisting medical problems ${ }^{[4]}$. Approximately one-third of the hospital mortalities occur in critically ill patients inside ICU ${ }^{[5]}$ are responsible for $10-20 \%$ of global hospital costs. Early identification of characteristics of critically ill patients requiring RICU and their suspected outcomes helps in improvement of these outcomes and reduction of their mortality rate ${ }^{[6]}$. Hence, the present study was conducted to study spectrum of pulmonary disease affecting the persons admitted to respiratory intensive care unit.

\section{Materials and methods}

The present study was conducted in the Department of Pulmonary Medicine of the Medial institute. The ethical approval for the study was approved from the ethical board of the institute before starting the study. For a study, a total of 100 patients with various respiratory diseases admitted to pulmonary ICU were selected. A written informed consent was obtained from everyone after verbally explaining them the study protocol. A complete demographic data for each patient was obtained including past medical history, past surgical history, family history, social history, history of present illness.

The statistical analysis of the data was done using SPSS version 11.0 for windows. Chi- 
square and Student's t-test were used for checking the significance of the data. A p-value of 0.05 and lesser was defined to be statistical significant.

\section{Results}

Table 1 shows demographic data of the participants. We observed that mean age of the participants was 51.65 years. The age of participating patients ranged between 30 to 70 years. The number of male subjects was 66 and number of female subjects was 34 [Fig 1]. Table 2: Distribution of patients according to various diagnosis. We observed that highest number of patients had bronchial asthma $(n=22)$, followed by chronic empyema $(\mathrm{n}=19)$, Pulmonary $\mathrm{Tb}$ $(n=16)$, COPD $(n=14)$, Pneumothorax $(n=12)$, pneumonia $(n=11)$. The lowest number of patients were seen with Bronchiectasis $(n=6)$. The results on comparing were found to be statistically non-significant. $(\mathrm{p}>0.05)$ [Fig 2]

Table 1: Demographic data of the participants

\begin{tabular}{|c|c|}
\hline Variables & Values \\
\hline Mean age (years) & 51.65 \\
\hline Age range (years) & $30-70$ \\
\hline Number of male subjects & 66 \\
\hline Number of female subjects & 34 \\
\hline
\end{tabular}

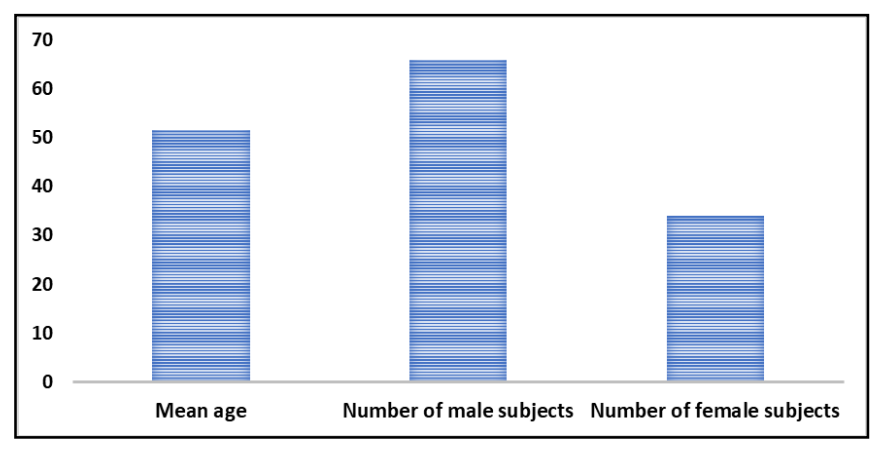

Fig 1: Demographic data of participants

Table 2: shows distribution of patients according to various diagnosis

\begin{tabular}{|c|c|c|}
\hline Diagnosis & No. of patients & Percentage \\
\hline Bronchial Asthma & 22 & 22 \\
\hline Bronchiectasis & 6 & 6 \\
\hline Chronic Empyema & 19 & 19 \\
\hline COPD & 14 & 14 \\
\hline Pneumonia & 11 & 11 \\
\hline Pneumothorax & 12 & 12 \\
\hline Pulmonary Tb & 16 & 16 \\
\hline Total & $\mathbf{1 0 0}$ & $\mathbf{1 0 0}$ \\
\hline
\end{tabular}

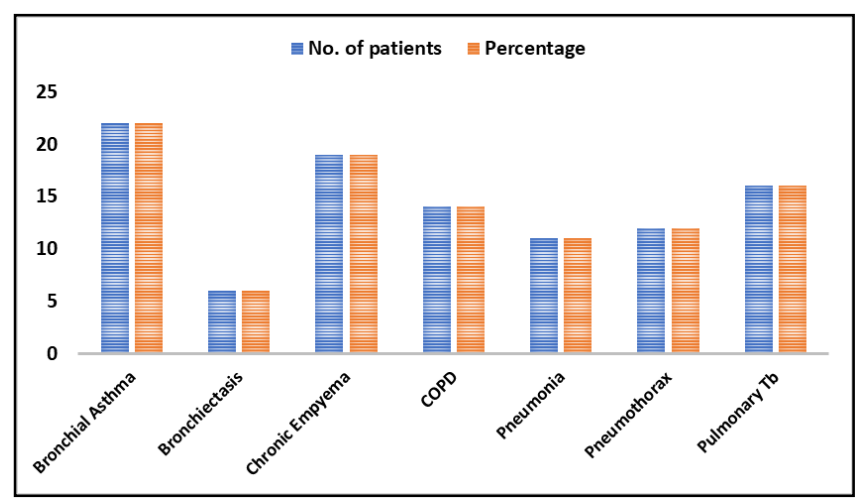

Fig 2: The results on comparing were found to be statistically nonsignificant. $(p>0.05)$

\section{Discussion}

In the present study, we observed that bronchial asthma was the most common disease among patients admitted to pulmonary ICU. Chronic empyema, Pulmonary Tb, COPD, Pneumothorax were also quite common among patients admitted to pulmonary ICU. The results on comparison were found to be statistically non-significant. The results were compared with previous studies from the literature and found to be consistent. Volakli $\mathrm{E}$ et al., investigated differences in the impact of respiratory tract and abdominal sites of infection on organ failure and survival. The SOAP study was a cohort, multicenter, observational study which included data from all adult patients admitted to one of 198 participating intensive care units (ICUs) from 24 European countries during the study period. In this substudy, patients were divided into two groups depending on whether, on admission, they had abdominal infection but no respiratory infection or respiratory infection but no abdominal infection. The two groups were compared with respect to patient and infection-related characteristics, organ failure patterns, and outcomes. Of the 3,147 patients in the SOAP database, 777 (25\%) patients had sepsis on ICU admission; $162(21 \%)$ had abdominal infection without concurrent respiratory infection and 380 (49\%) had respiratory infection without concurrent abdominal infection. Age, sex, and severity scores were similar in the two groups. On admission, septic shock was more common in patients with abdominal infection who were also more likely to have early coagulation failure and acute renal failure. In contrast, patients with respiratory infection were more likely to have early neurological failure. The median length of ICU stay was the same in the two groups, but the median length of hospital stay was longer in patients with abdominal than in those with respiratory infection. ICU (29\%) and hospital (38\%) mortality rates were identical in the two groups. They concluded that there are important differences in patient profiles related to the site of infection; however, mortality rates in these two groups of patients are identical. Mohan CK et al., studied the predictors of mortality in patients with respiratory infection in Indian patients as there is scarce data. A prospective observational study was done in a tertiary care center from August 2017 to February 2018. Patients admitted to ICU with acute respiratory infection were included in the study. Data on demographic variables such as age, gender, diagnosis, and reason for ICU admission were recorded. We also noted the presence of comorbidities. Modified CPI score was calculated on day 1 and day 2. Cox regression univariate and multivariate analysis along with Kaplan Meier analysis was done for assessing factors associated with mortality. 303 patients satisfied the inclusion criteria and were included in the study. The mean age of the cohort was $56.05 \pm 16.37$ years and $62 \%$ were men. The most common diagnosis was Pneumonia (66\%) followed by COPD (43.5\%). Mean duration of hospital stay was $7.29 \pm 3.76$ days. The mortality rate was $17.8 \%$. On multivariate Cox regression analysis CPI score $>4$ on day 1 , CPI score $>6$ on day 2 , number of antibiotics $>2$ were independently associated with increased hazard for mortality. They concluded that modified CPI score $>4$ on day 1 , CPI score $>6$ on day 2 , Usage of $>2$ antibiotics were independently associated with increased hazard for mortality in patients admitted to ICU with a respiratory infection ${ }^{[7,8]}$. 
Saydain G et al., conduceted a retrospective study describing the clinical course of 38 patients with idiopathic pulmonary fibrosis (IPF) admitted to the intensive care unit (ICU). There were 25 males and 13 females who were the mean age of $68.3 \pm 11.5$ years. Twenty patients were on corticosteroids at the time of admission to the hospital, and 24 had been on home oxygen therapy. The most common reason for ICU admission was respiratory failure. The Acute Physiology and Chronic Health Evaluation III-predicted ICU and hospital mortality rates were $12 \%$ and $26 \%$, whereas the actual ICU and hospital mortality rates were $45 \%$ and $61 \%$, respectively. They did not find significant differences in pulmonary function or echocardiogram findings between survivors and nonsurvivors. Mechanical ventilation was used in 19 patients (50\%). Sepsis developed in nine patients. Multiple organ failure developed in 14\% of the survivors and in $43 \%$ of the nonsurvivors. Ninety-two percent of the hospital survivors died at a median of 2 months after discharge. These findings suggest that patients with IPF admitted to the ICU have poor short- and longterm prognosis. Patients with IPF and their families should be informed about the overall outlook when they make decisions about life support and ICU care. Ghoneim AH et al., determined the admission pattern and outcome of patients in the Respiratory Intensive Care Unit (RICU) of Zagazig University Hospitals, Egypt. All cases admitted to RICU during the period from March 2010 to October 2010. They were 200 cases $\{126$ males $(63 \%)$ and 74 females $(37 \%)\}$ with an age range from 11 to 86 years. They were classified according to the causes of admission to RICU into 162 cases due to primary respiratory causes $(81 \%)$ and 38 cases due to secondary respiratory causes (19\%). On admission the following were carried out for all patients: full medical history, chest examination, assessment of Glasgow Coma Scale (GCS) and Acute Physiology and Chronic Health Evaluation II (APHCHE II) score, arterial blood gases analysis, plain chest and heart X-ray, computerized tomography (CT) electrocardiography (ECG) or echocardiography (ECHO) study when needed and assessment of the outcome. Two hundred cases were admitted during the study period: $57 \%$ were referred by chest physicians, $14.5 \%$ from other hospitals, $13.5 \%$ from other departments and others from chest ward and emergency room (ER). The mean GCS and APHACHE II score were $12.7 \pm 3.97$ and $14.4 \pm 6.5$ respectively. The length of stay in RICU was 7.2 \pm 7.4 days. Analysis of outcome of the cases showed that 70 patients (35\%) were transferred to chest ward, 61patients $(30.5 \%)$ died and 54 patients $(27.0 \%)$ were discharged to home. There was a significant difference between cases with primary (1ry) and secondary (2ry) respiratory causes regarding outcome with mortality rate $(26.6 \%)$ among cases with 1ry respiratory causes while in cases with 2 ry respiratory causes were $60.4 \%$. Outcome as regards source of admission showed that the highest percentage of death occurred among cases referred from chest ward and non chest physicians $(63.7 \%$ and $62.5 \%$ ) respectively. There was a significant association between outcome and duration of stay. Concerning the outcome on using mechanical ventilation, the mortality rate in mechanically ventilated patients was $52.05 \%$ while in non mechanically ventilated patients it was $47.5 \%$. This study showed that the best prognosis of admitted patients to RICU was for those who were transferred earlier especially those transferred by chest physicians and patients with 1ry respiratory diseases than those with 2ry respiratory diseases. Also, cases with high Glasgow Coma Scale and low APACH II score and those with a short duration of stay in RICU, especially without the need for mechanical ventilation had a good prognosis. Therefore, considering those aspects in the clinical practice would be reflected as a better outcome on dealing with RICU patients ${ }^{[9,10]}$.

\section{Conclusion}

Within the limitations of the present study, it can be concluded that bronchial asthma, chronic empyema, Pulmonary Tb, COPD and Pneumothorax are most common disease among pulmonary admitted patients.

\section{References}

1. Bolaji BO, Kolawole IK. The Intensive Care Unit of the University Teaching Hospital, Ilorin, Nigeria: a ten year review (1991-2001) S. Afr. J Anesth. Analg, 2005, 146-150.

2. David A. Gruenberg, Wayne Shelton Influencing length of stay in the ICU Am. J Crit. Care. 2006; 15:502-509.

3. Afessa B1, Morales IJ, Scanlon PD, Peters SG. Prognostic factors, clinical course, and hospital outcome of patients with chronic obstructive pulmonary disease admitted to an intensive care unit for acute respiratory failure. Crit Care Med. 2002; 30(7):1610-5.

4. Ghoneim A, Hussein R, El-Ghamry R, Mahmoud L. Patterns of admitted cases to Respiratory Intensive Care Unit at Zagazig University Hospitals, Egypt. EJCT 2013; 62:661-668.

5. Friedrichs J, Wilson G, Chant C. Long-term outcomes and clinical predictors of hospital mortality in very long stay intensive care unit patients. Crit Care. 2006; 10:R59.

6. Moreno R, Agthe D. ICU discharge decision-making: are we able to decrease post-ICU mortality? Intensive Care Med. 1999; 25:1035-1036.

7. Volakli E, Spies C, Michalopoulos A, Groeneveld AB, Sakr Y, Vincent JL. Infections of respiratory or abdominal origin in ICU patients: what are the differences? Crit Care. 2010; 14(2):R32. doi:10.1186/cc8909

8. Mohan CK, Mahandra M. Predictors of mortality in patients with respiratory infection admitted to ICU in a tertiary care centre. J Pulmon. 2019; 3(1):4-7.

9. Saydain G, Islam A, Afessa B, Ryu JH, Scott JP, Peters SG. Outcome of Patients with Idiopathic Pulmonary Fibrosis Admitted to the Intensive Care Unit. American Journal of Respiratory and Critical Care Medicine, Volume 166, Issue 6.

10. Ghoneim AH, Hussein RM, Lamia RG, Mahmoud Y. Patterns of admitted cases to Respiratory Intensive Care Unit at Zagazig University Hospitals, Egypt. Egyptian Journal of Chest Diseases and Tuberculosis. 2013; 62(4):661-668. 